\title{
Temporal Behavior of MIMO Channel Quality Metrics
}

\author{
Ping-Heng Kuo and Peter J. Smith \\ Department of Electrical and Computer Engineering, \\ University of Canterbury, \\ Christchurch, New Zealand \\ \{phk15,p.smith\}@elec.canterbury.ac.nz
}

\begin{abstract}
The performance of MIMO systems can be improved or optimized if channel state information (CSI) is known at the transmitter. In such systems, the transmitter adapts to the channel based on some channel metric(s). The design and performance of the adaptive scheme depends on the timevarying nature of the metric(s). Some previous work in this area [1] includes a derivation of level crossing rates (LCR) and average fade durations (AFD) for channel eigenvalues. In this paper, we extend this work to study the temporal behavior of three channel metrics. In particular, we derive the approximate LCR and AFD for the eigenvalue sum, eigenvalue range and simultaneous eigenmode outages. The analysis has been verified through simulations.
\end{abstract}

\section{INTRODUCTION}

Due to the potential benefits of Multiple Input Multiple Output (MIMO) systems, there has been intense research in the area of optimizing MIMO system performance and data rates, via various applications of power allocation and/or adaptive techniques based on eigenchannels (also known as eigenmodes) [2]. To achieve the desired gain, most proposed schemes need a feedback link (unless the system is TDD based) to allow channel diagonalization at the transmitter and therefore a realization of the eigenchannels [3]. However, implementation of feedback is not practical in some scenarios due to the extra resources required.

Nevertheless, feedback control schemes can be more feasible in practice if the temporal behavior of the channel is known, since appropriate adaptation rates can be evaluated. In previous work, approximate formulas for level crossing rates (LCR) and average fade durations (AFD) of individual eigenvalues have been derived [1]. Many adaptive algorithms, however, are based on the overall channel condition, instead of individual eigenstreams.

Here we identify three different channel metrics that convey useful information about overall channel status:

- eigenvalue sum,

- eigenvalue range,

- simultaneous eigenvalues outages.

The physical importance of these three metrics is described below.

The eigenvalue sum is equivalent to the trace of the channel correlation matrix or the squared Frobenius norm, which is an important parameter of the system. It is well-known that, when the power is uniformly allocated at the transmitter, the eigenvalue sum indicates the effective channel gain (as in space-time block coding schemes [4]). In general, a larger eigenvalue sum indicates better SNR performance. Thus, some proposed schemes have used it as a benchmark for adaptive control. For example, an antenna selection algorithm based on the eigenvalue sum is given in [5]. Also, in [6], a bit-allocation algorithm over eigenmodes is discussed. In such schemes, a higher bit budget will be given when the eigenvalue sum is large. Thus, how the eigenvalue sum evolves over time is an important issue for system design.

The eigenvalue spread [7] is another useful indicator of channel state. Generally, under the assumptions of equal power allocation and high SNR, more capacity can be attained if the eigenvalues are less spread out. In the low SNR region, however, the optimal policy is to allocate power to the strongest eigenmode only, so a larger eigenvalue spread is more desirable [8]. Also, the gain of water-filling (compared to uniform power allocation) increases with the difference between the largest and smallest eigenvalue due to lower power wastage [9]; Hence we consider this eigenvalue difference as a possible channel metric and term it the eigenvalue range or eigenvalue distance. The usual application of such a metric is in switching between two modes of operation, i.e. between water-filling and uniform power allocation.

Finally, some schemes have combined STBC and eigenbeamforming to exploit advantages from both categories $[10][11][12]$. In such cases, the same information is conveyed on multiple eigenmodes in order to obtain diversity gains. However, when the gains of all the eigenmodes used drop below their minimum required levels simultaneously, the system experiences an "outage". Hence we investigate how frequently such simultaneous outages occur.

For all three channel metrics, we compute approximate LCRs and AFDs for the baseline channel model, where the channel coefficients are independent, identical distributed (i.i.d) Rayleigh. The rest of the paper is organized as follows: Section II describes the MIMO channel model and eigenvalue statistics. The approximate LCR, AFD and simulations for the three channel metrics are presented in Sections III, IV and V respectively. Finally, we conclude in Section VI.

\section{Channel Model and Literature Review}

Consider a $\left(N_{T}, N_{R}\right)$ MIMO system with $N_{T}$ transmit and $N_{R}$ receive antennas. We postulate both transmitter and receiver antenna arrays are located in rich-scattering environments and the channel is assumed to be flat fading, so the propagation channel $\boldsymbol{H}$ is an $N_{R} \times N_{T}$ matrix that 
contains i.i.d. complex Gaussian entries with zero mean and unit magnitude variance (Rayleigh fading). Here we define $m=\min \left(N_{R}, N_{T}\right), n=\max \left(N_{R}, N_{T}\right)$, and the $m \times m$ Wishart matrix $W$ is given by

$$
\boldsymbol{W}=\left\{\begin{array}{ll}
\boldsymbol{H} \boldsymbol{H}^{\dagger}, & \text { for } N_{R} \leq N_{T} \\
\boldsymbol{H}^{\dagger} \boldsymbol{H}, & \text { for } N_{T}<N_{R}
\end{array} .\right.
$$

where $(\cdot)^{\dagger}$ denotes Hermitian transpose. Using the Singular Value Decomposition (SVD), $\boldsymbol{H}$ can be transformed into a set of parallel scalar channels. The ordered eigenvalues of $\boldsymbol{W}\left(\lambda_{1} \geq \ldots \geq \lambda_{m}\right)$ can be regarded as power gains of $m$ independent spatial channels intrinsic in the multiple antenna link [8]. The joint density function of these eigenvalues is provided in [13]:

$$
\begin{aligned}
f\left(\lambda_{1}, \ldots, \lambda_{m}\right)= & \prod_{i=1}^{m}[(n-i) !(m-i) !]^{-1} \exp \left(-\sum_{i=1}^{m} \lambda_{i}\right) \\
& \times \prod_{i=1}^{m} \lambda_{i}^{n-m} \prod_{i<j}\left(\lambda_{i}-\lambda_{j}\right)^{2} .
\end{aligned}
$$

The marginal density of any individual eigenvalue can be obtained by serial integration since the the joint density is simply a polynomial with exponential terms. Remarkably, the marginal density of all eigenvalues can be approximated extremely well by a gamma distribution for small systems, and mixtures of gamma distributions for larger systems [14][1]. Furthermore, the LCR formula for a gamma process across a threshold $T$ has been successfully derived in [1]:

$$
\begin{aligned}
\operatorname{LCR}_{G}(T)= & \frac{(\theta T)^{k-1 / 2} \sqrt{-R^{\prime \prime}(0)} \exp (-\theta T)}{\sqrt{\pi} \Gamma(r)} \\
& \times \Phi(k-r, k-r+1 / 2, \theta T)
\end{aligned}
$$

where $\Phi(a, b, c)$ is the confluent hypergeometric function; $r$ and $\theta$ are shape and scale parameters that can be calculated from the first two moments of the gamma process; $k$ is chosen to be the smallest integer greater or equal to $r . R^{\prime \prime}(0)$ is the second derivative of the autocorrelation function (ACF) of the underlying Gaussian process at $\tau=0$ [1]. By approximating the eigenvalue process with a gamma process and assuming that the temporal behavior of the independent channel matrix entries are governed by the Jakes process [15], the LCR for individual eigenvalues has been approximated in [1] as

$$
\begin{aligned}
\operatorname{LCR}_{\lambda}(T)= & \frac{(\theta T)^{k-1 / 2} \pi f_{D} \sqrt{2(1-K)} \exp (-\theta T)}{\sqrt{\pi} \Gamma(r)} \\
& \times \Phi(k-r, k-r+1 / 2, \theta T) .
\end{aligned}
$$

where $f_{D}$ is the maximum Doppler shift and $K$ is a casedependent constant that need to be calculated. More details are given in [1].

\section{LCR FOR EIGENVALUE SUM}

Since the eigenvalue sum, denoted $\gamma$, is equivalent to the trace of a Wishart matrix, it is in fact a sum of $2 N_{R} N_{T}$ independent $\chi_{1}^{2}$-distributed random variables. Hence $\gamma$ is $\chi^{2}$ distributed with $2 N_{R} N_{T}$ degrees of freedom [13]. The probability density function (p.d.f) of $\gamma$ is given by [16]:

$$
f(\gamma)=\frac{\gamma^{N_{R} N_{T^{\prime}}-1} \exp (-\gamma)}{\Gamma\left(N_{R} N_{T}\right)}
$$

where $\Gamma(\cdot)$ is the gamma function.

By utilizing the LCR formula for a $\chi^{2}$ process in [17], the LCR formula for the eigenvalue sum can be given by

$$
\operatorname{LCR}_{\gamma}(T)=\frac{\pi^{-1 / 2} \sqrt{-R^{\prime \prime}(0)} T^{N_{R} N_{T}-1 / 2} \exp (-T)}{\Gamma\left(N_{R} N_{T}\right)}
$$

As in [17], $R^{\prime \prime}(0)$ is the second derivative of the autocorrelation function (ACF) of the underlying Gaussians of the $\chi^{2}$ process at $\tau=0$. As we have assumed the channel is varying according to the Jakes process, the ACF is $J_{0}\left(2 \pi f_{D} \tau\right)$, where $J_{0}(\cdot)$ symbolizes the modified zero-order Bessel function of first kind. Thus, $R^{\prime \prime}(0)$ is found to be $-2 \pi^{2} f_{D}^{2}$. Substituting in (6) a closed-form formula for the LCR of the eigenvalue sum is acquired:

$$
\operatorname{LCR}_{\gamma}(T)=\frac{\sqrt{2 \pi} f_{D} T^{N_{R} N_{T}-1 / 2} \exp (-T)}{\Gamma\left(N_{R} N_{T}\right)}
$$

The AFD follows trivially since

$$
\operatorname{AFD}_{\gamma}(T)=\frac{\operatorname{Prob}(\gamma<T)}{\operatorname{LCR}_{\gamma}(T)}
$$

Figs.1-2 illustrate simulation results for the LCR and AFD of the eigenvalue sum for $(4,4)$ and $(2,8)$ cases respectively, and show excellent agreement with equations (7) and (8).

For scenarios involving correlated Rayleigh channels, the eigenvalue sum is $\chi^{2}$-distributed with dependent components. Here it is possible to write down the eigenvalue sum LCR by applying the appropriate results in [18]. Numerical computation and the resulting LCR appears to be difficult and is beyond the scope of this paper.

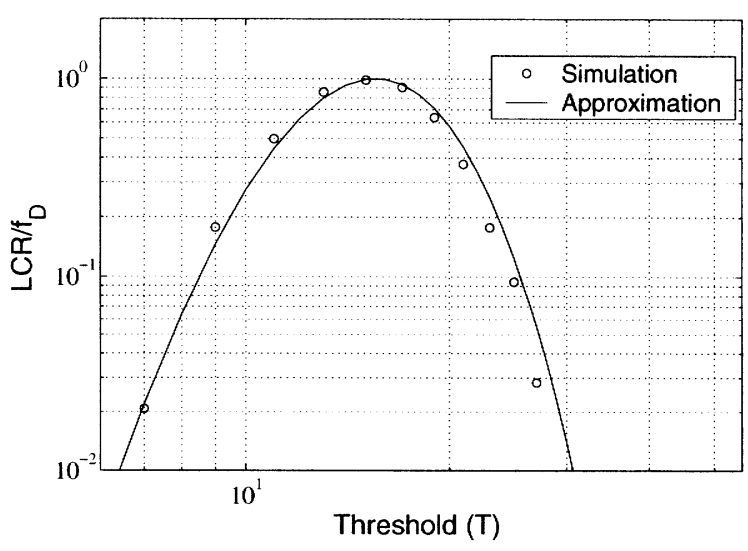

Fig. 1. The LCR of eigenvalue sum for a $(4,4)$ system. 


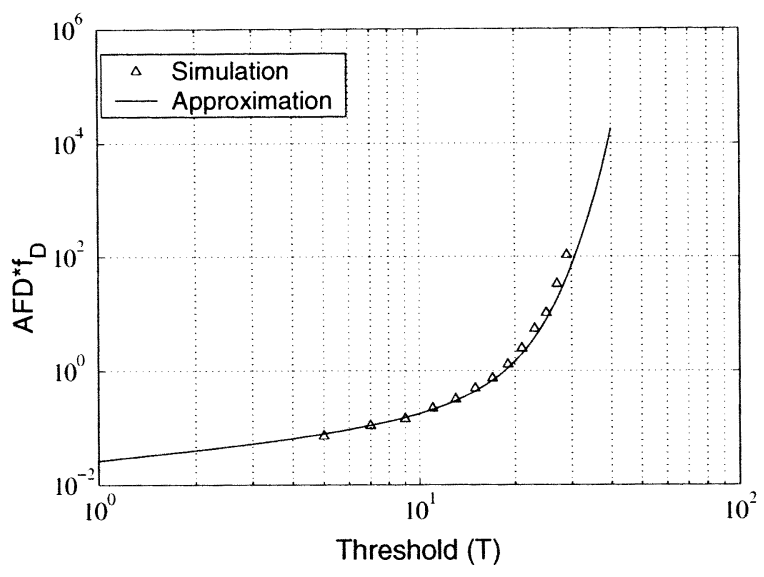

Fig. 2. The AFD of eigenvalue sum for a $(2,8)$ system.

\section{LCR FOR EIGENVALUE RANGE}

To measure the eigenvalue spread, the condition number $\left(\lambda_{1} / \lambda_{m}\right)$ is sometimes used. We prefer to consider the eigenvalue range in this work as the condition number can have infinite mean when $N_{R}=N_{T}$. Related results in [19][20] show that

$$
\mathrm{E}\left(\frac{1}{\lambda_{m}}\right)=\frac{1}{N_{R}-N_{T}}
$$

which demonstrates the tendency of the inverse minimum eigenvalue to become long tailed. Hence we consider the eigenvalue range, defined as $u=\lambda_{1}-\lambda_{m}$. In order to proceed analytically, we require the p.d.f of the eigenvalue range $u$.

Firstly, we integrate the joint density function of the eigenvalues (2) serially, to obtain the joint density function of the largest and smallest eigenvalue $\left(f\left(\lambda_{1}, \lambda_{m}\right)\right)$ only. Then, replace $\lambda_{m}$ by $\left(\lambda_{1}-u\right)$, and the joint density function of $\lambda_{1}$ and $u$ is acquired.

$$
f\left(\lambda_{1}, u\right)=\left.f\left(\lambda_{1}, \lambda_{m}\right)\right|_{\lambda_{m}=\lambda_{1}-u}
$$

Finally, we obtain the marginal density of $u$ by integration over $\lambda_{1}$. Due to space constraints, we only show the p.d.f of $u$ for the $(4,4)$ case as an example:

$$
\begin{aligned}
f_{u_{(4,4)}} & (u)=\frac{1}{3} e^{-3 u} u^{5}+5 e^{-3 u} u^{2}-5 e^{-u} u^{3} \\
& -\frac{1}{144} e^{-2 u} u^{8}+e^{-2 u} u^{4}+2 e^{-3 u} u^{4}+5 e^{-u} u^{2} \\
+ & \frac{1}{48} e^{-u} u^{6}+2 e^{-u} u^{4}-10 e^{-2 u} u^{2}+5 e^{-3 u} u^{3} \\
& -\frac{1}{3} e^{-u} u^{5}+\frac{1}{48} e^{-3 u} u^{6}-\frac{1}{8} e^{-2 u} u^{6}
\end{aligned}
$$

Since it has been shown that each eigenvalue can be approximated by a gamma variable [14], we extrapolate and propose approximating the eigenvalue range, $u$, by a gamma. This hypothesis is verified by fitting $u$ by a gamma for $(3,3)$ and $(4,4)$ cases using the method of moments. For instance, the first two moments of $u$ for the $(4,4)$ case are 9.5223 and 98.2506, so $\operatorname{Var}(u)=7.5767$. Using the method of moments we fit the gamma distribution with shape parameter $r=11.9675$ and scale parameter $\theta=1.2568$. In Fig. 3 we show the exact

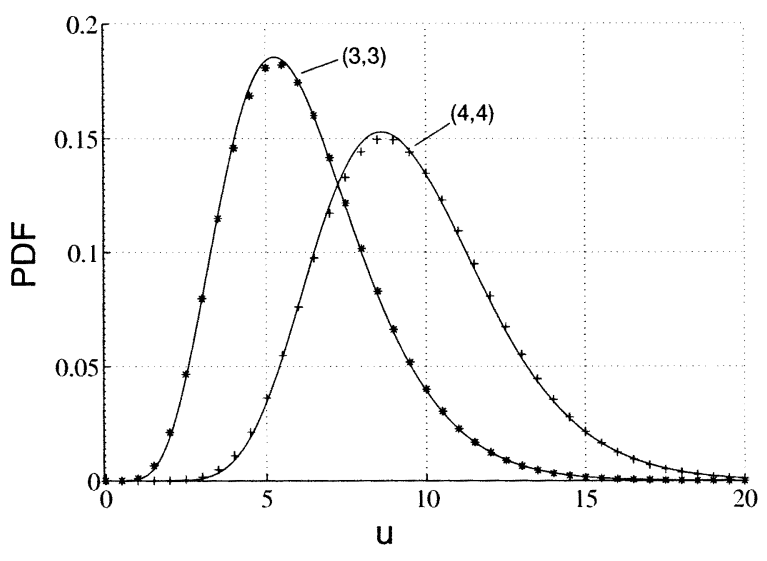

Fig. 3. p.d.f of $u$ in $(3,3)$ and $(4,4)$ systems; solid line represents the exact p.d.f., points denote the gamma approximation.

distribution and the gamma approximation for the $(3,3)$ and $(4,4)$ cases.

We can therefore approximate the LCR by applying (3) directly. Since first and second moments can be calculated exactly, $r$ and $\theta$ can be computed for use in (3). The only remaining parameter needed is $R^{\prime \prime}(0)$. To find the ACF, we utilize a certain stochastic differential equation (SDE) derived by Bru [21]. This equation was modified in [1] to apply to the eigenvalues of Wishart matrix $\boldsymbol{W}$ where the elements of $\boldsymbol{H}$ follow the Jakes process. The resulting SDE is

$$
d\left(t \lambda_{i}(t)\right)=\left(n+S_{i}(t)\right) d t+2 \sqrt{t \lambda_{i}} d B_{i}(t)
$$

where

$$
S_{i}(t)=\sum_{k \neq i} \frac{\lambda_{i}(t)+\lambda_{k}(t)}{\lambda_{i}(t)-\lambda_{k}(t)},
$$

the $\lambda_{i}(t)$ are the time varying eigenvalues and $B_{i}(t)$ is the driving Brownian motion process of the SDE. Since $u=\lambda_{1}-$ $\lambda_{m}$, we have $d u=d \lambda_{1}-d \lambda_{m}$, and hence (12) can be extended to form a new SDE for $u$ :

$$
\begin{array}{r}
d(t u(t))=\left(S_{1}(t)-S_{m}(t)\right) d t \\
+2 \sqrt{t \lambda_{1}(t)} d B_{1}(t)-2 \sqrt{t \lambda_{m}(t)} d B_{m}(t)
\end{array}
$$

Using the standard Euler approximation, the SDE above is approximated for small time incrementss, $\tau$, by

$$
\begin{array}{r}
(t+\tau) u(t+\tau)-t u(t) \approx\left(S_{1}(t)-S_{m}(t)\right) \tau \\
+2 \sqrt{t \lambda_{1}(t)}\left(B_{1}(t+\tau)-B_{1}(t)\right) \\
-2 \sqrt{t \lambda_{m}(t)}\left(B_{m}(t+\tau)-B_{m}(t)\right)
\end{array}
$$

Multiplying (15) by $u(t)$ an taking expectation gives, after a little algebra:

$\mathrm{E}[u(t) u(t+\tau)] \approx \frac{t}{t+\tau} \mathrm{E}\left[u^{2}(t)\right]+\frac{\tau}{t+\tau} \mathrm{E}\left[u(t)\left(S_{1}(t)-S_{m}(t)\right)\right]$

The normalized ACF for $u(t)$ is therefore

$R_{u}(\tau) \approx \frac{\frac{t}{t+\tau} \mathrm{E}\left[u^{2}(t)\right]+\frac{\tau}{t+\tau} \mathrm{E}\left[u(t)\left(S_{1}(t)-S_{m}(t)\right)\right]-\mathrm{E}[u(t)]^{2}}{\operatorname{Var}[u(t)]}$ 
As in [1] we must look at the particular time point $t=$ $\tau\left[J_{0}\left(2 \pi f_{D} \tau\right)^{-2}-1\right]^{-1}$ in (17) for the SDE to match the case of interest, where elements of $\boldsymbol{H}$ follow the Jakes process. This gives

$$
R_{u}(\tau) \approx 1+\frac{2 \pi^{2} f_{D}^{2} \tau^{2} \mathrm{E}\left[u(t)\left(S_{1}(t)-S_{m}(t)\right)-u(t)^{2}\right]}{\operatorname{Var}[u(t)]}
$$

Following [1] the ACF of the underlying Gaussian process is $R(\tau)=\sqrt{R_{u}(\tau)}$ and so we obtain

$$
R^{\prime \prime}(0)=\frac{2 \pi^{2} f_{D}^{2} \tau^{2} \mathrm{E}\left[u(t)\left(S_{1}(t)-S_{m}(t)\right)-u(t)^{2}\right]}{\operatorname{Var}[u(t)]}
$$

The value of $R^{\prime \prime}(0)$ is calculated and then applied in the gamma LCR formula (3), which results in a good fit to the simulated eigenvalue range LCR. The comparison between analytical results and simulations $(3,3)$ and $(4,4)$ cases are shown below.

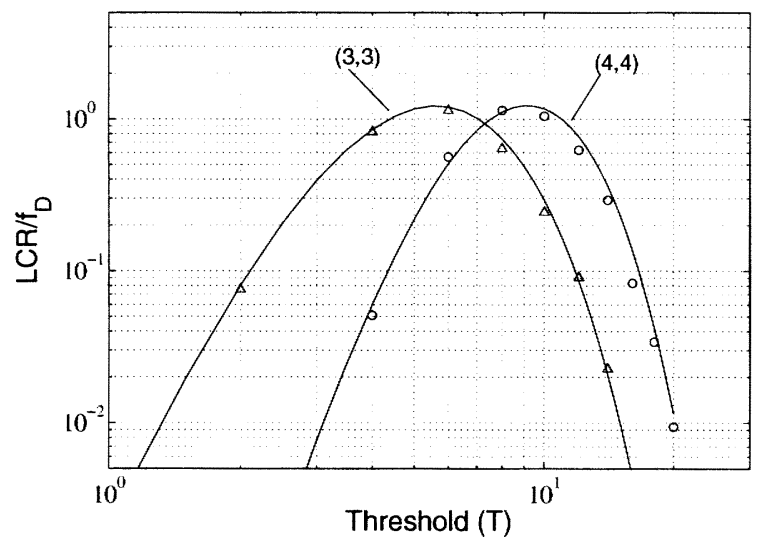

Fig. 4. The LCR of eigenvalue range for $(3,3)$ and $(4,4)$ systems. Solid line represents analytical approximations and points denote simulation results.

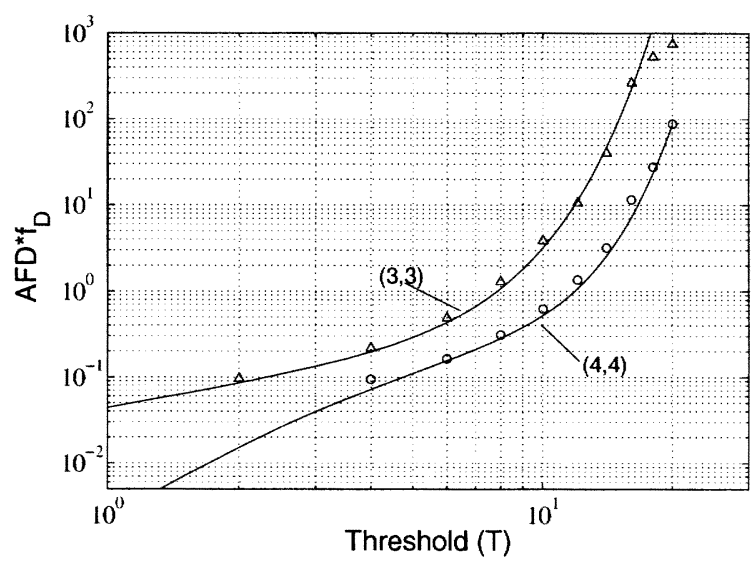

Fig. 5. The AFD of eigenvalue range for $(3,3)$ and $(4,4)$ systems. Solid line represents analytical approximations and points denote simulation results.

\section{Simultaneous OUTAGE RATE FOR EIGENVALUES}

It is assumed that the $m$ eigenmodes inherent in a MIMO system can be crudely approximated as $m$ independent random processes in parallel. One may assign arbitrary threshold levels (different or identical) on each of them to indicate outage. We say the system enters the "outage" state when none of the eigenmodes is above the assigned threshold. To approximate how often such a situation occurs, the simultaneous crossing rate of the eigenmodes is required. In fact, this occurs whenever an eigenmode down-crosses its threshold while all other eigenmodes are already below their own thresholds. Hence, we can express the simultaneous crossing rate as follows:

$$
\begin{array}{r}
\operatorname{LCR}_{\lambda_{1}, \ldots, \lambda_{m}}\left(T_{\lambda_{1}}, \ldots, T_{\lambda_{m}}\right) \\
=\sum_{i=1}^{m} \operatorname{LCR}_{\lambda_{i}}\left(T_{\lambda_{i}}\right) \prod_{k \neq i}^{m} \operatorname{Prob}\left(\lambda_{k}<T_{\lambda_{k}}\right)
\end{array}
$$

Note this is virtually an application of the formula derived in [22], since eigenmodes can be viewed as multiple links configured in parallel. The formula needs the LCR for individual eigenvalues, which is readily available (4). For the simultaneous AFD, which measures how long on average that multiple eigenvalues stay below their thresholds simultaneously, it is straightforward to show that:

$$
\operatorname{AFD}_{\lambda_{1}, \ldots, \lambda_{m}}\left(T_{\lambda_{1}}, \ldots, T_{\lambda_{m}}\right)=\frac{\prod_{k=1}^{m} \operatorname{Prob}\left(\lambda_{k}<T_{\lambda_{k}}\right)}{\operatorname{LCR}_{\lambda_{1}, \ldots, \lambda_{m}}\left(T_{\lambda_{1}}, \ldots, T_{\lambda_{m}}\right)}
$$

The simulation results for 2-transmit or 2-receive antenna systems $(m=2)$ are presented below. We select a set of arbitrary pairs of thresholds for the two eigenvalues. For ease of demonstration, we only show selected LCR and AFD results for $(2,2)$ and $(2,4)$ systems (Figs. 6-9). The agreement between analytical approximations and simulated values are roughly acceptable. Note that the approximation is motivated by the fact that the eigenvalues, although not independent, are only weakly correlated.

\section{CONCLusions}

The temporal behavior of MIMO channel metrics is important for system design. In this paper, we have presented methods for calculating approximate LCRs and AFDs for three different MIMO channel metrics. Firstly, the $\chi^{2}$-distribution nature of the eigenvalue sum leads to a simple LCR formula, which only requires a knowledge of the number of antennas, Doppler frequency and threshold level. Next, we analyzed the first order statistics of the eigenvalue range, and derived its autocorrelation in order to calculate LCR. Finally, a crude approximation to the simultaneous crossing rate for multiple eigenvalues is given. All these results seem to be promising due to their agreement with simulations. Nonetheless, some future work may be needed to improve their accuracy, or to investigate time-variations of other channel metrics. 


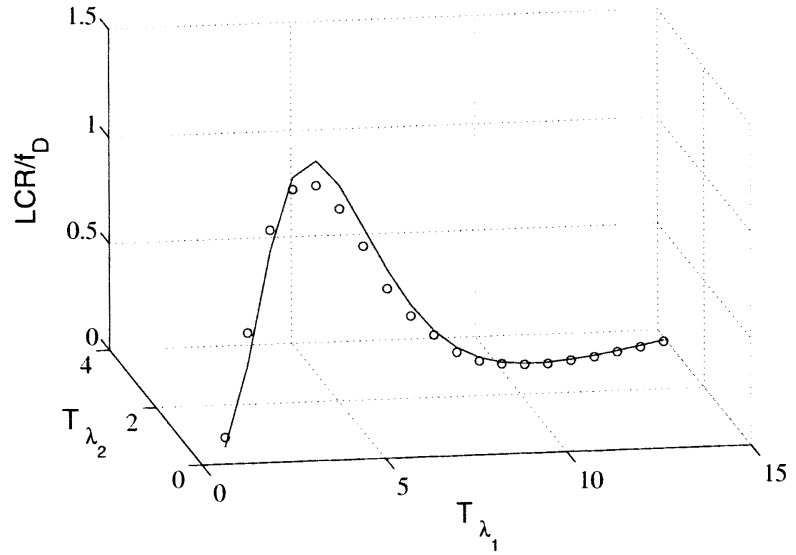

Fig. 6. The simultaneous LCR of both eigenvalues in a (2,2) system. Solid line represents analytical approximations and points denote simulation results.

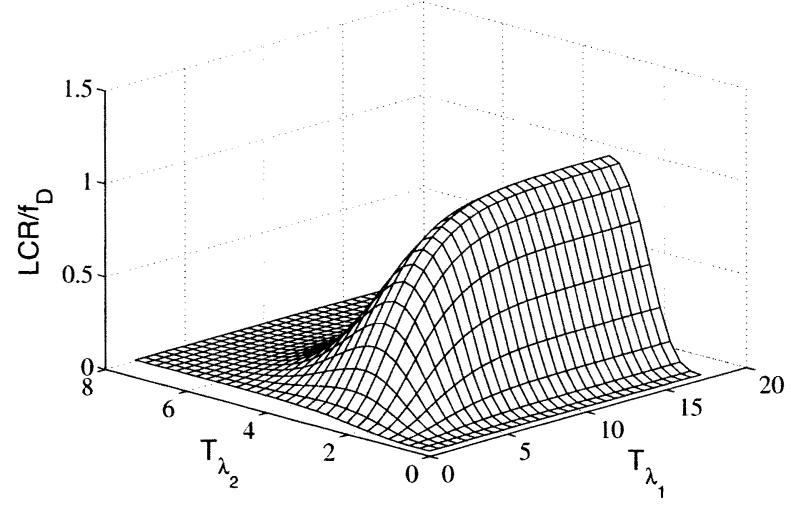

Fig. 7. The approximate simultaneous LCR for two eigenvalues in a $(2,4)$ system. The mesh plot shows LCR at any arbitrary pair of threshold levels.

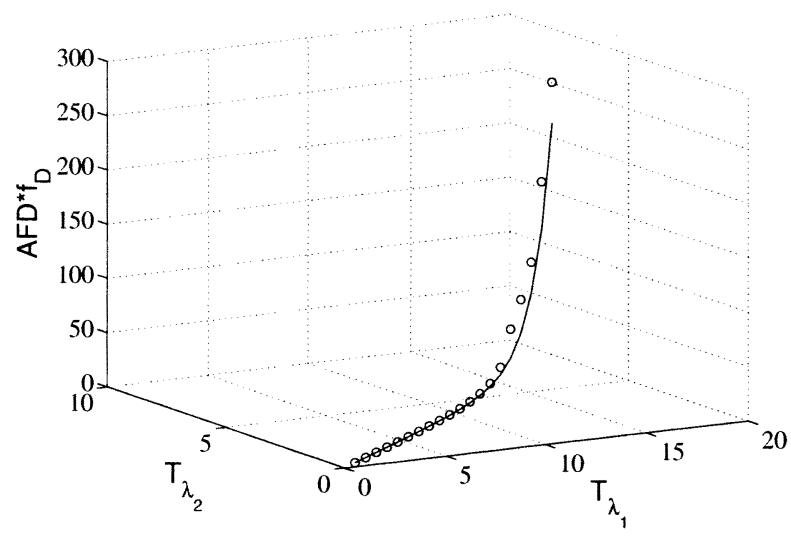

Fig. 8. The simultaneous AFD of both eigenvalues in a $(2,4)$ system. Solid line represents analytical approximations and points denote simulation results.

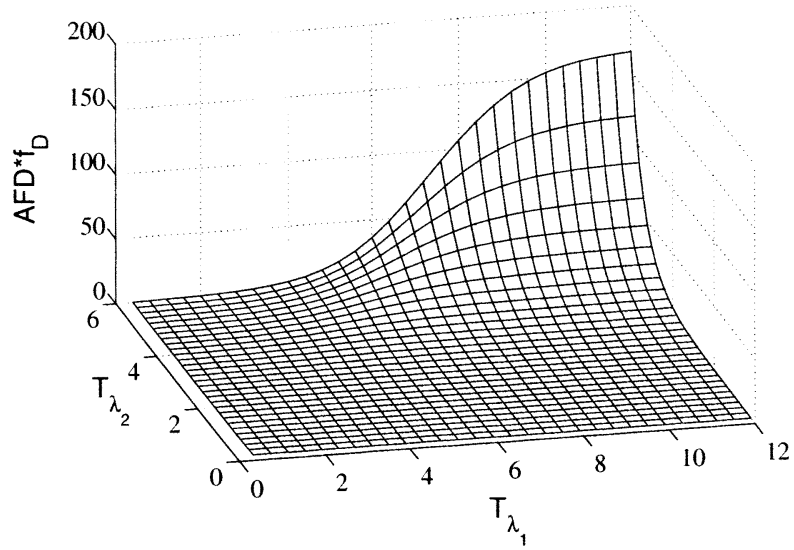

Fig. 9. The approximate simultaneous AFD for two eigenvalues in a $(2,2)$ system. The mesh plot shows AFD at any arbitrary pair of threshold levels.

\section{REFERENCES}

[1] P. J. Smith, P.-H. Kuo, and L. M. Garth, "Level crossing rate for MIMO channel eigenvalues: implications for adaptive systems," Proc. IEEE ICC., May 2005, to be published.

[2] J. B. Andersen, "Array gain and capacity for known random channels with multiple elemenrt arrays at both ends," IEEE J. Select. Areas Commun., vol. 18, pp. 2172-2178, Nov. 2000.

[3] G. G. Raleigh and J. M. Cioffi, "Spatio-temporal coding for wireless communication," IEEE Trans. Commun., vol. 46, pp. 357-366, Mar. 1998.

[4] S. Sandhu and A. Paulraj, "Space-time block codes: A capacity perspective," IEEE Commun. Lett., vol. 4, pp. 384-386, Dec. 2000

[5] T. Shu and Z. Niu, "A near-optimal antenna selection in MIMO system by using maximum total eigenmode gains," in Proc. IEEE GLOBECOM, 2003, pp. 297-301.

[6] K. Miyashita, T. Nishimura, T. Ohgane, Y. Ogawa, Y. Takatori, and $\mathrm{K}$. Cho, "High data-rate transmission with eigenbeam-space division multiplexing (E-SDM) in a MIMO channel," in Proc. IEEE VTC Fall, 2002, pp. 1302-1306.

[7] K. Sulonen, P. Suvikunnas, J. Kivinen, L. Vuokko, and P. Vainikainen, "Study of different mechanisms providing gain in MIMO systems," in Proc: IEEE VTC Fall, 2003, pp. 352-356.

[8] D. Tse and P. Viswanath, Fundamentals of Wireless Communication. EECS224b Course Textbook, University of California-Berkeley, 2004.

[9] P. Almers, F. Tufvesson, O. Edfors, and A. F. Molisch, "Measured capacity gain using water-filling in frequency selective MIMO channels," in Proc. IEEE PIMRC, 2002, pp. 1347-1351.

[10] G. Jongren, M. Skoglund, and B. Ottersten, "Combining beamforming and orthogonal space-time block coding," IEEE Trans. Inform. Theory, vol. 48, pp. 611-627, Mar. 2002.

[11] S. Zhou and G. B. Giannakis, "Optimal transmitter eigen-beamforming and space-time block coding based on channel mean feedback," IEEE Trans. Signal Processing, vol. 50, pp. 2599-2613, Oct. 2002.

[12] A. Pascual-Iserte, A. I. Perez-Neira, and M. A. Lagunas, "A maximin approach for robust MIMO design: Combining OSTBC and beamforming with minimum transmission power requirements," in Proc. IEEE ICASSP, vol. 2, May 2004, pp. 1-4.

[13] A. Edelman, "Eigenvalues and condition numbers of random matrices," Ph.D. dissertation, MIT, Cambridge, 1989.

[14] J. T. Y. Ho and P. J. Smith. "A quick simulation method for fading communications channels using a novel eigenvalue importance sampling technique," in Proc. IEEE VTC Fall, 2002, pp. 449-453.

[15] W. C. Jakes, Microwave Mobile Communications. Piscataway, NJ: IEEE Press Classic Reissue, 1995.

[16] M. Wennstrom, M. Helin, A. Rydberg, and T. Oberg, "On the optimality and performance of transmit and receive space diversity in MIMO channels," in Proc. IEE Seminar on MIMO, Dec. 2001, pp. 4/1-4/6.

[17] R. A. Silverman, "The fluctuation rate of the chi process," IRE Trans. Inform. Theory, vol. 4, no. 1, pp. 30-34, Mar. 1958. 
[18] G. Lindgren, "Slepian models for $\chi^{2}$-processes with dependent components with application to envelope upcrossings," J. Appl. Prob., vol. 26, pp. 36-49, 1989.

[19] E. Jorswieck, G. Wunder, V. Jungnickel, and T. Haustein, "Inverse eigenvalue statistics for Rayleigh and Rician MIMO channels," in Proc. IEE Seminar on MIMO, Dec. 2001, pp. 3/1-3/6.

[20] A. M. Tulino, A. Lozano, and S. Verdu, "Bandwidth-power tradeoff of multiple-antenna systems in the low-power regime," DIMACS Series in Discr. Math. and Theo. Comp. Sci, vol. 62, pp. 15-42, 2003.

[21] M. F. Bru, "Diffusions of perturbed principal component analysis," $J$. Multivariate Anal., vol. 29, pp. 127-136, 1989.

[22] L. Yang and M.-S. Alouini, "Level crossing rate over multiple independent random processes - an extension of the applicability of the Rice formula," in Proc. IEEE GLOBECOM, 2003, pp. 1644-1648. 